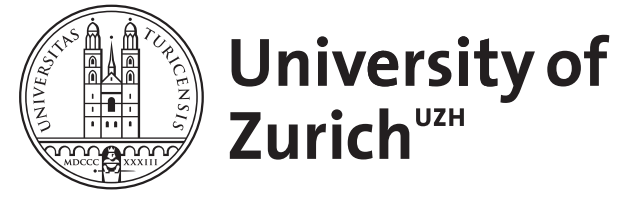
Archive

University of Zurich

University Library

Strickhofstrasse 39

CH-8057 Zurich

www.zora.uzh.ch

Year: 2011

Eine neue karische Mauerinschrift aus Kaunos

Kunnert, U ; Schürr, D ; Zingg, E

DOI: https://doi.org/10.1515/KADMOS.2010.010

Posted at the Zurich Open Repository and Archive, University of Zurich

ZORA URL: https://doi.org/10.5167/uzh-48571

Journal Article

Published Version

Originally published at:

Kunnert, U; Schürr, D; Zingg, E (2011). Eine neue karische Mauerinschrift aus Kaunos. Kadmos, 49(1):169-180.

DOI: https://doi.org/10.1515/KADMOS.2010.010 
Ursula KunNert - Diether SchÜrR - Emanuel ZingG

\title{
EINE NEUE KARISCHE MAUERINSCHRIFT AUS KAUNOS
}

\author{
Der archäologische Kontext \\ (U. Kunnert - E. Zingg)
}

An der Außenseite der Stadtmauer von Kaunos konnte im Sommer 2010 eine karische Mauerinschrift aufgenommen werden, die von Bernhard Schmaltz 2006 entdeckt worden war. ${ }^{1}$ Sie findet sich auf einem als Binder dienenden Bossenquader der nördlichen Westmauer, etwa $20 \mathrm{~m}$ südlich der Pforte nördlich von Sivrihisar, einige Meter nördlich des Abschnitts, in welchem der Wehrgang mitsamt den Zinnen noch erhalten ist. Die Mauer ist hier aus dem in der Nähe anstehenden blaugrauen Marmor erbaut (Abb. 1). ${ }^{2}$

Der Bossenquader mit der Inschrift befindet sich in der ersten Lage über dem heutigen Gehniveau. Er ist $0,48 \mathrm{~m}$ hoch und $0,58 \mathrm{~m}$ breit, seine Dicke nicht messbar. Wie B. Schmaltz bei der Untersuchung der Stadtmauer festgestellt hat, nimmt in diesem Abschnitt die Schichthöhe nach oben hin ab. Da das Gelände ansteigt, bilden die niedrigen Mauerschichten nach einer gewissen Strecke hangaufwärts die Sockelzone. Um wieder zu den großen Blöcken der Sockelzone wechseln zu können, werden die niedrigen Mauerschichten auf komplizierte Weise mit den hohen verzahnt. Die dadurch entstehenden Nahtstellen sind im Mauerverlauf gut sichtbar. Dabei zeigt sich, dass sie sich meist nicht in voller Mauerhöhe von oben nach unten ziehen, sondern auch nur wenige Schichten erfassen konnten. Deshalb müssen diese Zäsuren nicht zwingend als Arbeitsabschnitte interpretiert werden, obschon dies in Einzelfällen denkbar ist. Der Block mit der Inschrift schließt eine Lage vor einer Zäsur ab. Nördlich anschlie-

\footnotetext{
1 Wir danken B. Schmaltz für den Hinweis auf die Inschrift sowie seine Auskünfte zur kaunischen Stadtmauer. C. Ișık und Chr. Marek haben uns freundlicherweise die Inschrift zur Bearbeitung überlassen. Für eine kritische Durchsicht danken wir ihm herzlich. Unser Dank gilt zudem U. Ruppe, der uns bei Fragen zum Bau von Stadtmauern behilflich war.

2 Die Koordinaten sind: N $36^{\circ} 49^{\prime}$ 57.77’, O 28 36’ 59.34”.
}

Kadmos Bd. 49, S. 169-180

(C) WALTER DE GRUYTER 2010

ISSN 0022-7498

DOI 10.1515/KADMOS.2010.010 


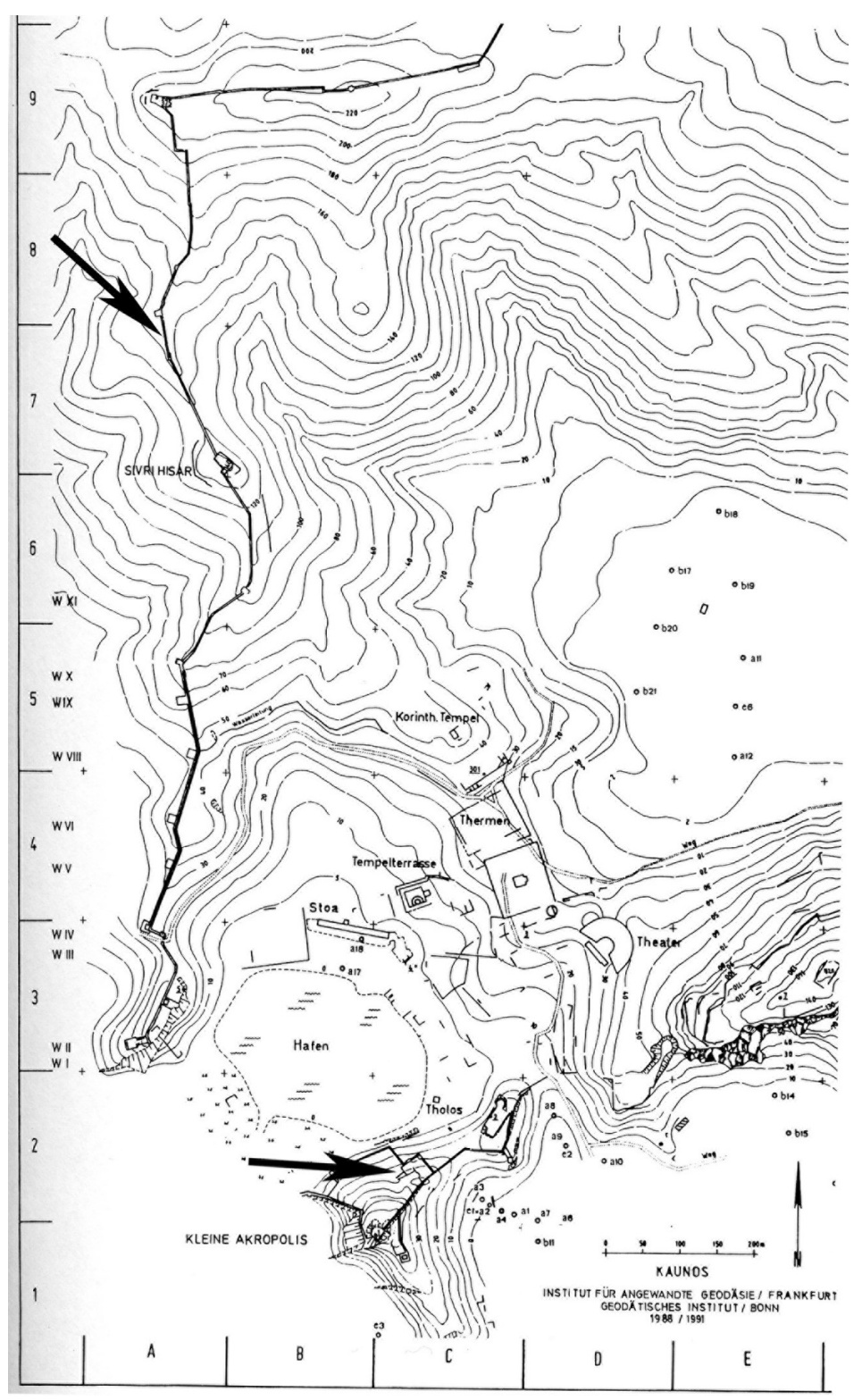

Abb. 1. Plan von Kaunos mit Markierung des Fundorts der neuen Mauerinschrift in A7 sowie der Mauerinschrift beim Demeterheiligtum in C2 (aus Schmaltz 1994, Abb. 1) 


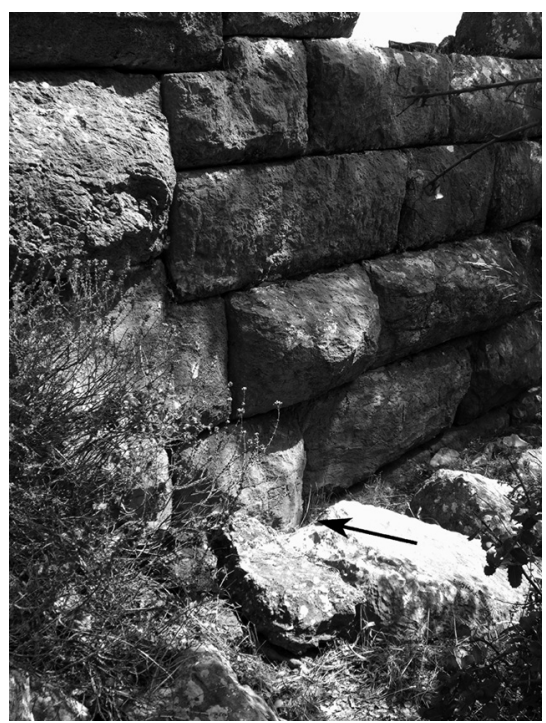

Abb. 2. Fundsituation. Die Nahtstelle ist in der linken Bildhälfte gut sichtbar. Der Pfeil weist auf den Block mit der Inschrift

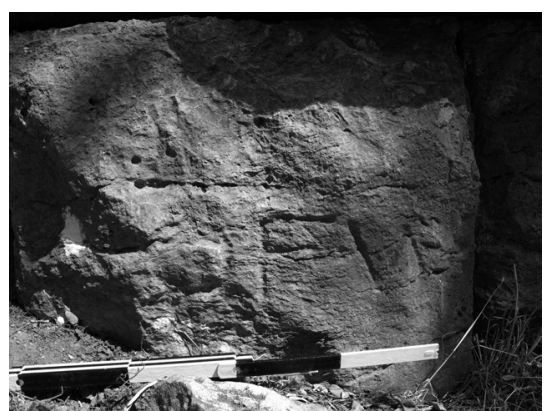

Abb. 3. Inschrift: OFИ orm ßend folgt ein großer Block der Sockelzone. ${ }^{3}$ Um wie viel tiefer das Gehniveau zum Zeitpunkt der Errichtung der Mauer lag, ist unklar (Abb. 2). ${ }^{4}$

Die Inschrift ist mittig auf der schmalen Außenseite des Binders platziert (Abb. 3). Die Buchstabenhöhe beträgt 0,07-0,08 m. Das Zeichen $И$ $m$ erweist eindeutig, dass eine Inschrift in karischem Alphabet vorliegt. Es scheint sich nicht um eine beiläufige Kritzelei zu handeln, da die Buchstaben relativ tief, einigermaßen sorgfältig und waagrecht eingehauen sind. Hinweise darauf, dass die Inschrift ursprünglich noch länger gewesen sein könnte, gibt es nicht. Es liegt keine sekundäre Verwendung des Inschriftträgers vor, da sich der Block weder im Material noch in der Bearbeitung von den umliegenden Blöcken unterscheidet.

Auf dem angrenzenden Läufer rechts ist ein senkrechter Strich ebenfalls etwa mittig und ungefähr gleich groß und gleich tief eingemeißelt. Der Abstand $\mathrm{zu}$ orm ist jedoch derart groß, dass ein unmittelbarer Bezug

\footnotetext{
Zu diesem Phänomen der nördlichen Westmauer vgl. B. Schmaltz 1994, 189f., zuletzt Schmaltz 2010, 318f.

4 Ohne Grabung sind wir nicht in der Lage zu bestimmen, wie hoch die Versturzlage von Mauerblöcken vor der Mauer ist. Unserem Eindruck nach dürfte jedoch die Differenz zum heutigen Bodenniveau gering sein, da nördlich des Quaders mit der Inschrift die großen Blöcke des Mauerwerks der Sockelzone noch ca. $2 \mathrm{~m}$ aufragen und darüber ursprünglich noch eine heute unbekannte Zahl niedriger Schichten aufging.
} 
nicht gegeben scheint. Im karischen Alphabet gibt es keinen Buchstaben, der nur aus einem senkrechten Strich besteht. Bei diesem Strich kann es sich um ein Symbol, ein griechisches Iota oder ein Zahlzeichen handeln.

$\mathrm{Da}$ in der karischen Epigraphik bisher weder Steinmetzzeichen noch Abkürzungen sicher belegt sind, gehen wir in den folgenden Ausführungen davon aus, dass die Inschrift aus einem Wort besteht.

Aufgrund der Buchstabenformen, die sich in das Spektrum der karischen Buchstaben aus Kaunos einfügen, lässt sich die neue Inschrift nicht datieren (s.u. Schrift und Sprache). Die jüngste sicher zu datierende karische Inschrift, C.Ka 5, die Bilingue von Kaunos, stammt vermutlich aus dem letzten Drittel des 4. Jh.s v. Chr. ${ }^{5}$

Es lässt sich nicht entscheiden, ob die Inschrift vor dem Einbau des Blockes in die Mauer oder danach angefertigt wurde. In letzterem Fall ist unklar, ob sie gleich nach dem Bau angebracht wurde und direkt mit ihm in Verbindung steht oder zu einem wesentlich späteren Zeitpunkt eingemeißelt wurde.

Schmaltz datiert den Mauerabschnitt, in welchem die neue Inschrift entdeckt wurde, in das letzte Viertel des 3. Jh.s v. Chr. Er stützt sich bei seiner Datierung auf Beobachtungen, die er an der südlichen Westmauer und der Südbastion der Kleinen Akropolis machte. Die dortigen Verstärkungen und Reparaturen bringt er in Zusammenhang mit einer Erdbebenkatastrophe im Jahr 227/6 v. Chr. Zeitgleich damit setzt er eine Erweiterung in sorgfältiger Quaderbauweise, zu welcher auch der Abschnitt mit der neuen Inschrift gehört. ${ }^{6}$

Falls Schmaltz' Datierung zutrifft, ergäbe sich daraus ein terminus post quem für die Inschrift, die damit einen sehr späten Beleg für die karische Schrift darstellen würde.

Weitere karische Buchstaben sind auf der heute sichtbaren Stadtmauer nicht entdeckt worden ${ }^{7}$, ihr Vorhandensein kann aber nicht völlig ausgeschlossen werden. Für die Interpretation von orm ist es nötig, karische und griechische Inschriften zu finden, die hinsichtlich des Anbringungsortes, der Länge und eventuell ihrer Deutung als Personenname (s.u. Schrift und Sprache) mit orm verglichen werden können.

\footnotetext{
Zu karischen Inschriften, die möglicherweise in das 3. Jh. v. Chr. datiert werden können, s. Schürr 2010a, 188f.; zur Datierung der Bilingue s. Marek 2006, 119 121.

Schmaltz 1991, 137-157; Schmaltz 1994, 189.

7 Vgl. Schmaltz 1991, 121-177; Schmaltz 1994, 185-204.
} 
Die einzige andere bekannte karische Mauerinschrift befindet sich an der Westseite einer Terrassenmauer des so genannten Demeterheiligtums in Kaunos ( pot $_{2} k o s ́ \lambda o a b[\dagger][\dagger]$ / ùa[†][; s.u. Schrift und Sprache). ${ }^{8}$ Diese ist ebenfalls auf nur grob bossierten Quadern, bei ihrer Entdeckung etwa in Hüfthöhe, und in nahezu gleichgroßen Zeichen (ca. 7,5 cm in der ersten Zeile, ca. $6 \mathrm{~cm}$ in der zweiten) eingemeißelt. Folgende Unterschiede lassen sich trotzdem feststellen: Die Inschrift beim Demeterheiligtum besteht aus mindestens 14 Zeichen, hat also wahrscheinlich mehr als nur einen Namen wiedergegeben. Sie wurde an einem zentralen Ort bei einem Heiligtum angebracht, wogegen orm an einem wenig zugänglichen Ort an der Feldseite der Stadtmauer eingemeißelt wurde. Karische Inschriften, die nur einen Personennamen im Nom. Sg. wiedergeben, sind aus Ägypten als Graffiti und auf Kleinfunden bekannt. ${ }^{9}$ Aufgrund ihres Anbringungsortes und ihrer Ausführungsqualität sind sie vom Zweck her mit der neuen Mauerinschrift nicht zu vergleichen.

Auch in der griechischen Epigraphik ist die Suche nach direkten Parallelen schwierig. Denkbar ist, dass derart kurze Mauerinschriften in der Forschung bisher zu wenig Beachtung gefunden haben und unpubliziert sind. ${ }^{10}$ Auf der Stadtmauer von Paestum findet sich eine Reihe von archaischen Inschriften, die in der jetzigen Anordnung teilweise nur einen Namen im Genitiv wiederzugeben scheinen. Ihr ursprünglicher Zweck ist heute nicht mehr sicher zu eruieren; vorgeschlagen wurde, hier Namen von Stiftern des Mauerbaus zu sehen. ${ }^{11}$ Die Untersuchung der griechischen Bauinschriften der klassischen und hellenistischen Zeit von Franz Georg Maier hat gezeigt, dass diese auch in der knappsten Form nicht nur den Namen des Stifters angeben. ${ }^{12}$ Noch ungeklärt ist die Frage nach der Bedeutung einer von den Buchstabenformen her wohl hellenistischen Inschrift an der SW-Ecke der Westtor-Bastion in Kaunos. Das heute erhaltene $\Delta[-$ ? $] /$ EП ergibt so keinen Sinn ${ }^{13}$ und unterscheidet sich hinsicht-

8 Marek 2006, K 5 = C.Ka 8; zum Fundort s. Abb. 1.

9 Beispiele bei Adiego 2007, 264f.

10 Die detailliert aufgenommenen Stadtmauern von Priene bieten nach der freundlichen Auskunft von U. Ruppe kein Beispiel.

11 Dunst 1966-67, 244-247, aus dessen Ausführungen nicht klar hervorgeht, ob die Blöcke bereits in ihrer ursprünglichen Anordnung in einer Stadtmauer vermauert waren.

12 Maier 1961, 29. Die kürzesten bei Maier aufgeführten Inschriften stammen von einem Turm auf Tenos (Bd. I, 1959, Nr. 39; 3./2. Jh. v. Chr., vgl. auch Nr. 40):

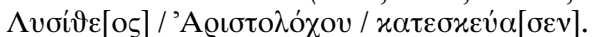

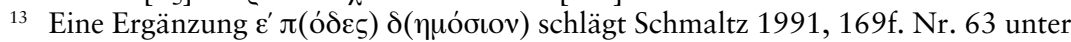
Verweis auf eine Inschrift auf Turm und Kurtine der Akropolis-Ostmauer in Nisy- 
lich des Anbringungsortes - einer gut sichtbaren Stelle in heute ca. $2 \mathrm{~m}$, ursprünglich wohl um die $3 \mathrm{~m}$ Höhe an einer Ausfallstraße der Stadt - von orm.

Die karische und griechische Epigraphik bietet demnach keine engen Parallelen zu der neu gefundenen karischen Inschrift. Nach den obigen Überlegungen ist eine Interpretation des Zwecks der Inschrift schwierig. Eine Deutung als Appellativum ist nicht völlig auszuschließen, doch spricht manches - wie im Folgenden (s.u. Schrift und Sprache) ausgeführt werden wird - für einen Personennamen im Nominativ Singular. Dies lässt in verschiedene Richtungen denken: Wenn die Inschrift zeitgleich mit dem Mauerbau anzusetzen ist, könnte orm den Namen eines am Bau beteiligten Handwerkers, Architekten oder Bauherrn, der für die Finanzierung der Mauer oder des Mauerabschnitts verantwortlich war, bezeichnen. Zur Angabe des Namens im Nom. Sg. hätte man sich in diesem Fall ein Verb wie $\chi \alpha \tau \varepsilon \sigma x \varepsilon v ́ \alpha \sigma \varepsilon v$ hinzuzudenken. Aus zwei Gründen scheint dieser Ansatz jedoch problematisch zu sein: Zum einen würde man bei einer offiziösen Signatur eine mindestens zweiteilige Namensformel, bestehend aus dem Individual- und dem Vatersnamen, erwarten. ${ }^{14}$ Zum anderen würde eine derartige Inschrift nahe eines markanten Punktes der Mauer angebracht sein, etwa bei einem Tor, einer Pforte oder einem Turm. Keinen Hinweis dafür, dass orm einen Bauleiter bezeichnen könnte, dürfte der Anbringungsort an einer Nahtstelle liefern, da diese, wie oben erwähnt, nicht unbedingt als Markierung von Bauabschnitten zu sehen ist. Die Nachbarschaft zur Nahtstelle dürfte daher zufällig sein. Wenn die Inschrift deutlich später als der Mauerbau eingemeißelt wurde, sind noch weitere Deutungen denkbar, die jedoch nicht mehr einzugrenzen sind.

\section{Schrift und Sprache \\ (D. Schürr)}

So kurz diese von B. Schmaltz bereits 2006 entdeckte Inschrift ist, sie steuert doch einiges zur Schriftgeschichte in Kaunos, zur Verwendung der karischen Schrift generell und zu unserer Kenntnis des Karischen bei.

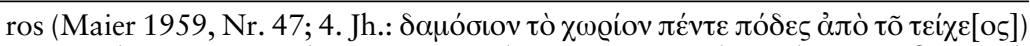
vor. Zu derartigen Regelungen zur Beachtung eines Bauabstandes an Außen- und/ oder Innenseiten von Stadtmauern aus Sicherheitsgründen in Mauerinschriften s. Winter 1971, 125 Anm. 60.

14 Vgl. etwa die Namensliste C.My 1 aus Mylasa. 
Was Zeichenformen betrifft, hat die Inschrift $o$ in etwa der gleichen Höhe wie die folgenden Zeichen, $r$ mit kürzerem unterem Querstrich (wie F), $m$ mit drei Schrägstrichen. Die Form des $r$ ist eine Besonderheit von Kaunos und findet sich auch auf den Stelenfragmenten $\mathrm{K} 2$ = C.Ka $2^{15}$ und $\mathrm{K} 3$ = C.Ka 4 - neben einer analogen Form des $\grave{u}$ mit kürzerem mittlerem Querstrich (wie E) - sowie in dem Graffito K 8 = C.Ka 6 - nur or - auf dem Fuß eines Kantharos, den Bernhard Schmaltz 1998, 209 „ungefähr um die Mitte des 4. Jh.s“ ansetzt. In der Bilingue K 1 = C.Ka 5 ist der untere Querstrich nur teilweise etwas kürzer, bei $\grave{u}$ der mittlere Querstrich „leicht kürzer“ (Frei-Marek 1997, 12). In diesen Fällen ist aber das o etwas (Stelenfragmente, Bilingue) bzw. deutlich (Graffito) kleiner. Anders ist das in der ersten Mauerinschrift vom sog. Demeterheiligtum K $5=$ C.Ka 8, wo $\grave{u}$ mit deutlich kürzerem Mittelquerstrich erscheint. Das - verglichen mit N - ,gewendete' $m$ ist für Kaunos charakteristisch, aber auf den Stelenfragmenten ist der erste Strich meist mehr oder weniger senkrecht. Mit drei Schrägstrichen wie hier erscheint $m$ in der Grabinschrift von Mezargediği K 4 = C.Ka 1, wo o so groß wie die anderen Zeichen ist, und in der Bilingue, außerdem wohl auch in dem Graffito auf dem rotfigurigen attischen Skyphosfragment PT-SK-15-8-2000 (4. Jh., eine genauere Datierung steht noch aus) von der sog. Palästraterrasse ${ }^{16}$. Der erste Strich beginnt zwar am Bruch, so dass ein vierter Strich ergänzt werden könnte (dann wäre $s$ zu lesen), aber der letzte Strich endet viel höher als der erste, was das Zeichen dem $m$ in der Felsgrabinschrift von Krya östlich von Kaunos (C.Kr 1) ähneln lässt. Das Graffito wird also ]mtì zu lesen sein, wobei das $\grave{u}$ hier einen sogar längeren mittleren Querstrich hat und alle Querstriche leicht aufwärts weisen.

15 Mit K werden die Inschriften von Kaunos nach Marek 2006 angeführt, alle karischen Inschriften mit den Siglen bei Adiego 2007 (wo C. für „Caria“ und E. für „Egypt“ steht), die Münzlegenden nach Konuk apud Adiego 2007. Die von Adiego 2007 eingeführten Änderungen der Umschrift übernehme ich aber nicht, siehe Schürr 2010b, 134f. Griechisch überlieferte karische Personen- und Ortsnamen werden nach Blümel 1992 bzw. 1998 ohne nähere Bezeichnung angeführt, griechisch überlieferte lydische und lykische Namen mit KPN nach Zgusta 1964. Für das Angebot, an der Publikation dieser Inschrift mitzuwirken, danke ich Christian Marek, für Auskünfte und Hinweise außerdem Winfried Held, Alexander Herda, Bernhard Schmaltz und Günter Vittmann.

16 Abb. 22 bei Piras 2009, 242. Abb. 21 bei Piras 2009, 242 scheint eine Art Monogramm zu zeigen, das m.E. nicht als karisch bestimmbar ist, Abb. 23 dürfte griechisch sein, da das vierte, durch eine Beschädigung verunklarte Zeichen sehr wahrscheinlich ein Pi ist (Hinweis von B. Schmaltz): Es ist also MYРП[ zu lesen. 
Trotz der Kürze fügt sich die neue Mauerinschrift also in das Spektrum der kaunisch-karischen Inschriften ein. Sie bietet aber eine Kombination von Zeichenformen, die sonst nirgends erscheint. Keine Unterschiede lassen sich gegenüber der Grabinschrift von Mezargediğ $i$ und der ersten Mauerinschrift erkennen. Nach den Zeichenformen würde man nicht vermuten, dass sie erst gegen Ende des 3. Jh.s geschrieben wurde. In Schürr 2010b, 188f. habe ich aber gezeigt, daß die Felsgrabinschrift C.Ka 3 in Kaunos nach der Form des $\breve{s}$ deutlich jünger sein dürfte als die Bilingue, so dass das Karische in Kaunos noch bis in das 3. Jh. geschrieben worden sein wird. Allerdings setzt Roos 1972, 42 das Grab wegen der darin gefundenen Keramik, vor allem wegen der Scherben eines rotfigurigen Kraters, „before the middle of the fourth century B.C.“ an.

Auch hinsichtlich des Lautbildes ist orm interessant, denn in der wachsenden Zahl der karischen Inschriften von Kaunos begegnet die Buchstabenfolge $o-r$ bemerkenswert häufig: in der Bilingue siebenmal, auf dem großen Stelenfragment viermal, in der Felsgrabinschrift K $10=$ C.Ka 3 und auf dem Kantharos je einmal. Das sind zusammen mit orm 14 Belege, und dazu kommt noch die Felsgrabinschrift in Krya, die auch in kaunischer Schrift und Sprache verfasst ist, mit zwei Belegen. Auch in den früheren karischen Inschriften Ägyptens ist $o-r$ oft vertreten ${ }^{17}$, und sogar in der bei Adiego 2007, 29 wiedergegebenen frühen Inschrift von Smyrna kommt es vor, aber unter den jüngeren Inschriften des übrigen Karien kehrt die Buchstabenfolge nur in einer einzigen Inschrift wieder, C.St 2 in Stratonikeia (siehe unten).

In den griechisch überlieferten karischen Ortsnamen ist das karisch $o-r$ entsprechende $\omega$ - $\varrho$ gut belegt, unter den Personennamen aber auffallenderweise kaum. In Lagina sind für denselben Mann im 4. Jh. v. Chr. die Schreibungen $\sum x o \alpha \varrho \alpha v o s$ und $\Sigma x \omega \varrho \alpha v o s$ belegt, wie

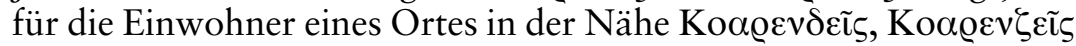

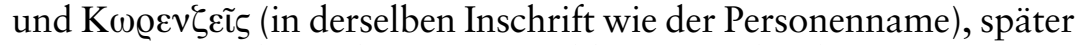

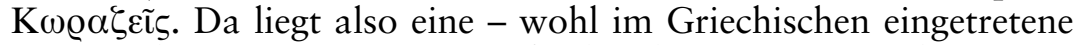
- Kontraktion o $\alpha>\omega \operatorname{vor}^{18}$. Außerdem kommt $\omega$ - $\varrho$ nur bei Personennamen in zwei verschiedenen Inschriften ebenfalls des 4. Jh.s in Mylasa vor (siehe unten).

$17 \mathrm{Zu}$ ihnen rechne ich auch die Bronzephialen C.Ha 1 und C.xx 1 mit der Objektbezeichnung orkn, siehe Schürr 2010b, 125.

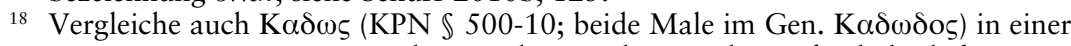
Liste von Männern aus Sardes aus dem 4. Jh. in Ephesos für lydisch katowas,

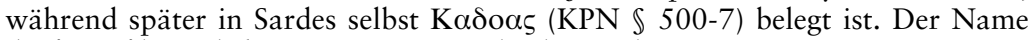
dürfte auf karisch kdou (E.Bu 1) zurückgehen (Schürr 2010a, 195). 
Die Häufigkeit von o-r in Kaunos könnte mit der vom übrigen Karischen abweichenden Lautentwicklung des Kaunischen zusammenhängen - und womöglich ein konservativer Zug sein? -, aber solange wir nicht einmal wirklich wissen, worauf das lange karische $o$ zurückgeht, muß das ein Verdacht bleiben.

Sogar die Buchstabenfolge $o-r-m$ ist in Kaunos nochmals belegt: auf dem großen Stelenfragment in Z. 10. Aber da scheint ouor wie in Z. 6 abtrennbar, so daß mit $m$ - wohl ein neues Wort beginnt. Plausibler ist, in Stratonikeia C.St 2, 6 šqem orm s[ und 7 šqed ormin $s[\mathrm{zu}$ segmentieren. Da dürfte an der ersten Stelle ein Passivpartizip und ein Nom. Sg. orm vorliegen, an der zweiten die 3. P. Prät. (?) Pl. des gleichen Verbs und ein Akk. Sg. ormñ. Orm könnte da ein Appellativum sein, und das käme natürlich auch bei der Mauerinschrift in Kaunos in Frage, so unklar es wäre, was es da für einen Sinn haben könnte.

Aber es ist wohl näherliegend, dass orm da ein Personenname ist, und das ist auch in Stratonikeia nicht ausgeschlossen. Anschließen könnte es in Kaunos dann an or auf dem Kantharos. Dieses hat Parallelen in Ägypten: Die Inschrift der Totenstele E.Me 41 beginnt mit 'orś' úpe, „des Ōr Stele“. Das Graffito E.Ab 15 in Abydos dürfte pdubẹ orś zu lesen und zu segmentieren sein: „Pedubēst, (Sohn) des Ōr“. Das Graffito E.Th 36 in Theben scheint unvollständig zu sein: 'orś, „(Sohn?) des Ōr“.

Günter Vittmann 2001, 42 hat nun in or einen ägyptischen Namen erkannt, d. h. einen als Personennamen gebrauchten Götternamen: „gewiß $\mathrm{Hr}$,Horus', gesprochen [hōr], der in der Spätzeit am häufigsten gebrauchte Personenname überhaupt“. Der Sohn in Abydos hat ebenfalls einen ägyptischen Namen: ,der von Bastet Gegebene'.

Einen weiteren Horusnamen * $J-H r-$ nicht belegt, aber nach einem in der Spätzeit belegten Muster gebildet - nimmt Vittmann 2001, 42 in aor an, auf der Totenstele E.Me 1 Vater des piub $[a] \xi i$,der der Bastet' und Großvater einer ttba ${ }^{\prime} i$, die von Bastet Gegebene'. In dem Graffito E.Bu 6 dürfte aor dagegen Vater eines puor sein, nach Vittmann 2001, 41 für äg. [puhór], der Hund', gräzisiert Пovw@ıs (u. ä.). Schließlich könnte noch isor auf der Totenstele E.xx 1 „mit dem häufigen Namen Ns-hr ,er/sie gehört dem Horus', gesprochen [əshōr], identisch sein “ (Vittmann 2001, 50). Vater und Sohn haben da karische Namen.

Danach sollte man annehmen, dass der or in Kaunos aus einer karomemphitischen Familie stammte, die nach Karien zurückgewandert und in Kaunos ansässig geworden war - falls er das Graffito 
selbst geschrieben hatte. Aber nun könnte orm, das nach Auskunft Vittmanns kein Horus-Name sein kann, nahelegen, in dem kaunischen or einen genuin karischen Namen zu sehen, homonym mit dem ägypto-karischen oder in Ägypten zur Wiedergabe von [họor] verwendet.

An einen genuin karischen Namen or ließe sich zunächst orou, wie mit Konuk OFOV auf den Münzen M51 und 52 (statt lykisch uwug) zu lesen ist, mit einem öfters belegten PN-Ausgang anschließen. Dieser Name könnte außerdem dem durch Stephanos von Byzanz

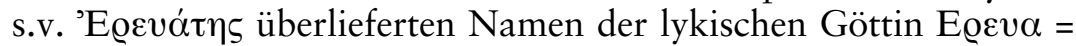

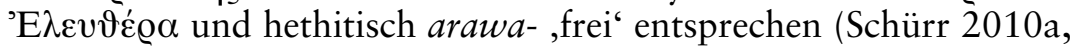
196). Zum Anschluß eines PN orm mit dem Suffix - $m$ lassen sich vergleichen:

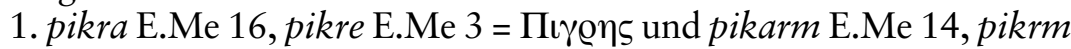

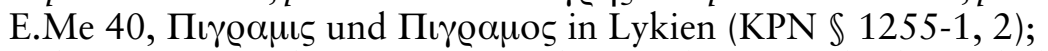

2. kúar E.Me 31, kuari E.Me 18a, kúri E.Th 34 (vergleiche auch die ONN Ko $\propto \varrho \omega v \delta / \alpha /$, Ko $\alpha \varrho \varepsilon v \delta / \alpha /$, beide bei Lagina, Kv $\alpha \varrho \delta \alpha$, Stadt Kariens nach Steph. Byz.) und der PN Kva@enos in Halikarnassos;

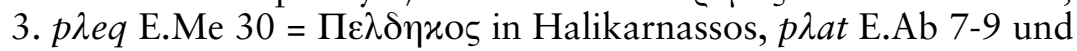

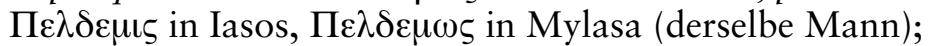

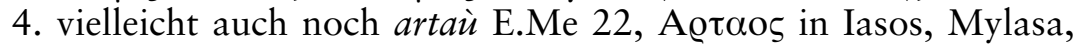

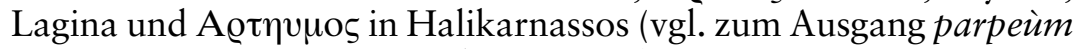
E.Me 25, paraeùm E.Me 8, beieùm E.Th 28).

Eine andere Erklärung legen aber die Fragmente zweier griechisch geschriebener karischer Personennamen in Mylasa nahe: [?] $\omega 0 \lambda \varepsilon \mu \iota \varsigma$ und Javwo@enos. Es liegt die Annahme nahe, dass zwischen beiden

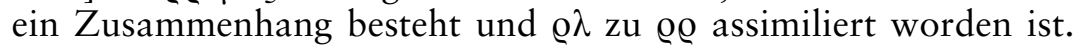
Dieser Lautwandel ist im Karischen tatsächlich belegt (Schürr 2010a, 200f.), allerdings gerade in Mylasa nicht. Man müsste also annehmen, dass der zweite Name fremder Herkunft ist. In Stratonikeia ist

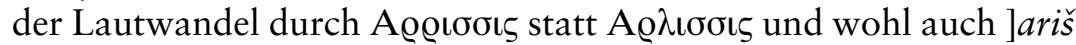
+ in C.St 1, 4 belegt, so dass dort orm die für - das dann vollständige $-\Omega \varrho \lambda \varepsilon \mu \iota \varsigma$ in Mylasa zu erwartende Form sein kann. Bei dem zweiten Namen dort liegt dann ein Kompositum, am ehesten mit dem auch sonst belegten Erstglied $\Pi \alpha v-$, nahe; theoretisch könnte man aber auch an eine Erweiterung von qanor in E.Th 34 denken, vergleiche dazu qan in E.Si 2. Aber bei qanor ist unklar, ob es sich um einen Namen handelt, und es ist natürlich auch ganz fraglich, ob $\Omega \varrho \lambda \varepsilon \mu \iota \varsigma$ eine Erweiterung von or ist.

Nun ist Assimilierung von $r l$ in Kaunos nicht belegbar und bei der vom übrigen Karischen deutlich abweichenden Lautentwicklung 
des Kaunischen (siehe vor allem das Fehlen von $e=[\overline{\mathrm{e}}]$ ) auch nicht unbedingt zu erwarten. Und wegen der Häufigkeit von $o-r$ in Kaunos wäre vielmehr zu erwarten, dass orm dort heimisch ist. Aber der Anschluß von orm in Kaunos an orm in Stratonikeia sowie $\Omega \varrho \lambda \varepsilon \mu \iota \varsigma$ und $\Pi$ ?] $\alpha v \omega \varrho \varrho \varepsilon \mu o \varsigma$ in Mylasa ist doch verlockend und damit auch die Bestimmung als Personenname, ob dieser nun in Kaunos heimisch war oder nicht.

Die neue Mauerinschrift besteht also vermutlich eher aus einem Personennamen als aus einem Appellativum - mehr lässt sich beim Stand unserer Kenntnis des Karischen nicht sagen. Und dabei ist zu berücksichtigen, dass wir über karische Personennamen viel mehr wissen als über den sonstigen Wortschatz. Die Kürze unterscheidet diese Inschrift auf jeden Fall von der ersten Mauerinschrift. Darin dürfte in der Sequenz pot ${ }_{2} k_{0}{ }^{\prime} \lambda o a b$ - (Mareks Lesung) ein Personenname auf $-\lambda o$ abzutrennen sein, vergleiche in Kaunos das frühe Graffito $\mathrm{K} 7$ = C.Ka 9, wo vermutlich urmio zu lesen und abzutrennen ist, außerdem auf der Bronzephiale C.Ha 1 psnio und im Heiligtum des Sinuri bei Mylasa auf der Basis C.Si 1 sñaid $\lambda_{0}$, vermutlich auch in der Steleninschrift C.Si 2, $4(\tilde{n} m-) a[.] \lambda o$ und in Halikarnassos A $\alpha \alpha \mu \mu \mu \varepsilon \lambda \delta \omega[\varsigma]$.

Am Beginn der Inschrift könnte außerdem eine Verbform auf - $t$ abzutrennen sein, denn auch die Inschriften E.Th 30 und E.Si 4 dürften mit einer solchen Verbform beginnen: bebint. Allerdings

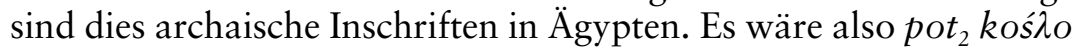
möglich, was immer dann folgt. Der Zusammenhang mit dem sog. Demeterheiligtum könnte für eine Votivinschrift sprechen.

\section{Bibliographie}

Adiego, I. J. 2007: The Carian Language, with an Appendix by Koray Konuk (= Handbook of Oriental Studies. Section 1: The Near and Middle East, vol. 68), Leiden/Boston.

Blümel, W. 1992: Einheimische Personennamen in griechischen Inschriften aus Karien, EpAnat 20, 7-34.

Blümel, W. 1998: Einheimische Ortsnamen in Karien, EpAnat 30, 163-184.

Dunst, G. 1966-67: Zu den Inschriften an der Stadtmauer von Paestum, RM 73-74, 244-247.

Frei, P. - Marek, Ch. 1997: Die karische Bilingue von Kaunos. Eine zweisprachige Staatsurkunde des 4. Jh.s v. Chr, Kadmos 36, 1-89.

Kalinka, E. 1901: Tituli Lyciae lingua Lycia conscripti (= Tituli Asiae Minoris I), Vindobonae. 
Maier, F. G. 1959-61: Griechische Mauerbauinschriften, 2 Bde., Heidelberg.

Marek, Ch. 2006: Die Inschriften von Kaunos (= Vestigia. Beiträge zur alten Geschichte 55), München.

Piras, D. 2009: Der archäologische Kontext karischer Sprachdenkmäler und seine Bedeutung für die kulturelle Identität Kariens, in: Rumscheid, F. (Hg.), Die Karer und die Anderen. Internationales Kolloquium an der Freien Universität Berlin, 13. bis 15. Oktober 2005, Bonn, 229-250.

Roos, P. 1972: The Rock-Tombs of Caunus 1. The Architecture (Studies in Mediterranean Archaeology 34:1), Göteborg.

Schmaltz, B. 1991: Kaunos 1988/89, Belleten 55, 121-177.

Schmaltz, B. 1994: Kaunos 1988-1991, AA, 185-234.

Schmaltz, B. 1998: Vorhellenistische Keramikimporte in Kaunos - Versuch einer Perspektive, Kadmos 37 (= Blümel, W. - Frei, P. - Marek, Ch. (Hgg.), COLLOQUIUM CARICUM. Akten der Internationalen Tagung über die karisch-griechische Bilingue von Kaunos, 31.10. - 1.11.1997 in Feusisberg bei Zürich), 203-210.

Schmaltz, B. 2010: Kaunische Mauern - zwischen Stil und Pragmatismus, in: van Bremen - Carbon 2010, 317-329.

Schürr, D. 2010a: Spätkarisch: Regionalisierung und Lautentwicklungen, in: van Bremen - Carbon 2010, 187-205.

Schürr, D. 2010b: Rezension von Ignacio J. Adiego, The Carian Language. Leiden/Boston 2007, Kratylos 55, 134-142.

van Bremen, R. - Carbon, J.-M. (Hgg.) 2010: Hellenistic Karia. Proceedings of the First International Conference on Hellenistic Karia - Oxford, 29 June - 2 July 2006 (= Ausonius Éditions, Études 28), Bordeaux.

Vittmann, G. 2001: Ägyptisch-Karisches, Kadmos 40, 39-59.

Winter, F. E. 1971: Greek Fortifications, London.

Zgusta, L. 1964: Kleinasiatische Personennamen, Prag. 